\title{
ROLAND BACHER
}

\section{YVES COLIN DE VERDIÈRE}

\section{Multiplicités des valeurs propres et transformations étoile-triangle des graphes}

Bulletin de la S. M. F., tome 123, no 4 (1995), p. 517-533

<http://www.numdam.org/item?id=BSMF_1995_123_4_517_0>

(C) Bulletin de la S. M. F., 1995, tous droits réservés.

L'accès aux archives de la revue «Bulletin de la S. M. F. » (http: //smf.emath.fr/Publications/Bulletin/Presentation.html) implique l'accord avec les conditions générales d'utilisation (http://www.numdam.org/ conditions). Toute utilisation commerciale ou impression systématique est constitutive d'une infraction pénale. Toute copie ou impression de ce fichier doit contenir la présente mention de copyright.

\section{Numdam}




\title{
MULTIPLICITÉS DES VALEURS PROPRES ET TRANSFORMATIONS ÉTOILE-TRIANGLE DES GRAPHES
}

\author{
PAR \\ Roland BACHER et Yves COLIN DE VERDIÈRE $\left(^{*}\right)$
}

\begin{abstract}
RÉSUMÉ. - On étudie l'invariant numérique $\mu \in\{0,1,2, .$.$\} des graphes finis défini$ dans un travail précédent du second auteur. Un tel graphe $\Gamma$ est dit $n$-critique si $\mu(\Gamma)=n$ et $\operatorname{si} \mu(\tilde{\Gamma})<n$ pour tout mineur strict $\tilde{\Gamma}$ de $\Gamma$. On montre que pour $n \geq 5$ une transformation étoile-triangle change un graphe $n$-critique en un autre graphe $n$ critique. On en déduit en particulier six nouveaux exemples de graphes 5 -critiques. Les bonnes propriétés de l'invariant $\mu$ par rapport aux transformations étoile-triangle permettent aussi de donner une preuve combinatoire du fait que $\mu$ (graphe planaire) $\leq 3$.

Abstract. - We show that the $\mu$-invariant of finite graphs introduced by Colin de Verdière behaves well under star-triangle transformations. This enables us for instance to find several new 5-critical graphs. Moreover, we use these star-triangle transformations in order to give a new proof of $\mu$ (planar graph) $\leq 3$.
\end{abstract}

\section{1. Énoncé des résultats}

Motivés par les calculs du premier auteur et les questions de R. Thomas, nous prouvons quelques résultats sur l'invariant $\mu$ d'un graphe fini connexe simple introduit dans [CV1], voir aussi [CV3]. Nos résultats portent sur le comportement de $\mu$ par les transformations étoile-triangle $Y \Delta$ et $\Delta Y$.

Une transformation $Y \Delta$ consiste à remplacer un sommet de valence trois et les trois arêtes le joignant à ses voisins $(Y)$ par un cycle formé de ces trois voisins et de trois nouvelles arêtes; de plus, s'il y a création d'arêtes doubles, on remplace chaque paire par une seule arête. La transformation

(*) Texte reçu le 17 février 1994, révisé le 24 octobre 1994.

Roland BACHER et Yves COLIN de Verdière, Institut Fourier, Université de Grenoble I, BP 74, 38402 Saint-Martin-d'Hères CEDEx (France).

Email : bacher@fourier.grenet.fr et ycolver@fourier.grenet.fr.

Classification AMS : 05 C 50, 47 A 10, 47 A 75.

BULLETIN DE LA SOCIÉTÉ MATHÉMATIQUE DE FRANCE 0037-9484/1995/517/\$5.00

(C) Société mathématique de France 
$\Delta Y$ relative à un cycle $\Delta$ de longueur 3 consiste à supprimer les trois arêtes du cycle et à joindre ses trois sommets à un nouveau sommet.
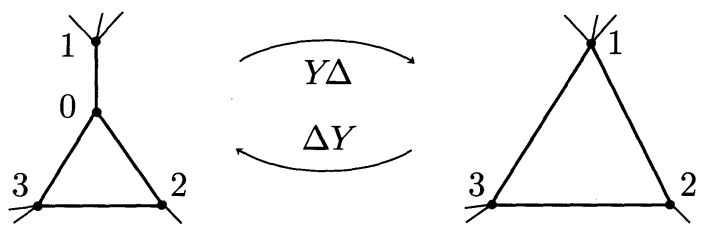

Figure 1. Les transformations $Y \Delta$ et $\Delta Y$.

Notons d'une part que la composition, dans cet ordre, d'une transformation $\Delta Y$ puis de la transformation $Y \Delta$ convenable est l'identité. Ceci ne vaut en général pas pour la composition dans l'autre ordre, comme on le voit par exemple en partant du graphe complet à 4 sommets. Notons d'autre part que les transformations $\Delta Y$ et $Y \Delta$ n'augmentent pas le nombre d'arêtes et préservent le nombre de composantes connexes; il en résulte que l'ensemble des graphes qu'on peut obtenir à partir d'un graphe $\Gamma$ donné par une succession de transformations $\Delta Y$ et $Y \Delta$ est un ensemble fini; voir par exemple la figure 2.

Introduisons aussi la transformation $* S$ (étoile-simplexe) par rapport à un sommet $s$ du graphe $\Gamma$. C'est une généralisation de la transformation $Y \Delta$ à des sommets qui ne sont pas de degré 3 . Elle consiste à relier tous les sommets voisins du sommet $s$ par des arêtes et à supprimer le sommet $s$ (ainsi que les arêtes entre $s$ et ses voisins). Ensuite on supprime d'éventuelles arêtes doubles.

Une transformation $* S$ portant sur un graphe $\Gamma$ est dite régulière si le graphe $\widetilde{\Gamma}$ obtenu en effaçant le sommet $s$ ainsi que ses sommets voisins (et toutes les arêtes incidentes en un de ces sommets) reste connexe.

Nos résultats principaux s'énoncent comme suit. Pour la définition de l'invariant $\mu$ et des graphes $n$-critiques, voir les rappels ci-dessous.

THÉORÈME 1. - Soit $\Gamma$ un graphe comme ci-dessus et soit $\Gamma^{\prime}=Y \Delta(\Gamma)$ un graphe obtenu à partir de $\Gamma$ par transformation $Y \Delta$; alors $\mu(\Gamma) \geq \mu\left(\Gamma^{\prime}\right)$.

THÉORÈmE 2. - Soit $\Gamma$ un graphe fini connexe simple et soit $s$ un sommet de degré $k$. Soit $\Gamma^{\prime}=* S(\Gamma)$ le graphe obtenue à partir de $\Gamma$ par transformation $* S$ en le sommet s. Si $\mu(\Gamma) \geq k+2$ on a $\mu(\Gamma) \leq \mu\left(\Gamma^{\prime}\right)$. Le résultat reste vrai si $\mu(\Gamma)=k+1$ et si la transformation $* S$ en $s$ est régulière.

THÉORÈmE 3. - Si $\Gamma$ est $n$-critique avec $n \geq 5$, alors tout graphe obtenu $\grave{a}$ partir de $\Gamma$ par une transformation $Y \Delta$ ou $\Delta Y$ est aussi $n$-critique.

TOME $123-1995-\mathrm{N}^{\circ} 4$ 
Le ThÉORÈme 3 s'applique par exemple au graphe complet à six sommets $K_{6}$. On trouve ainsi sept graphes 5-critiques représentés à la figure 2 .
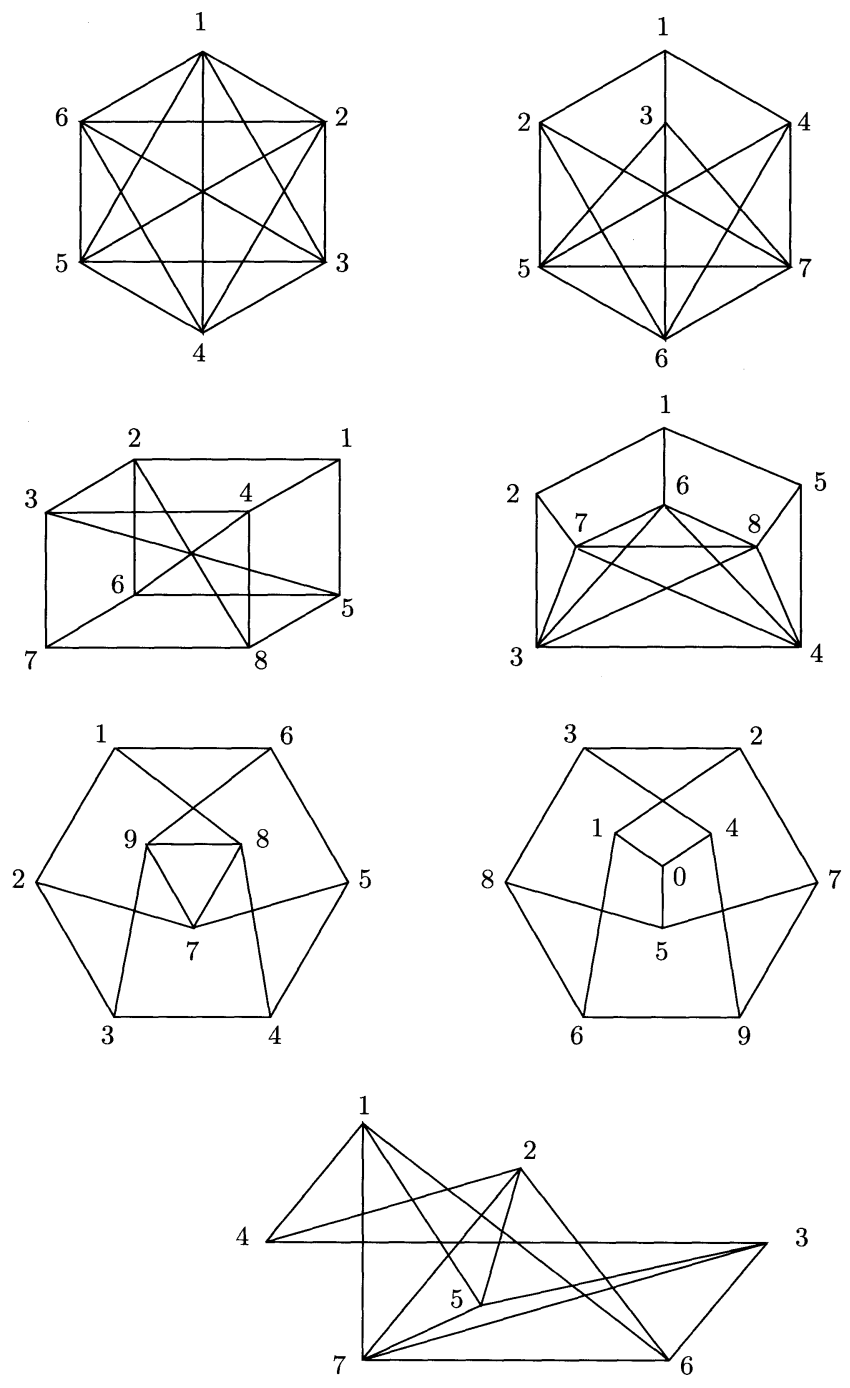

Figure 2. Les sept graphes obtenus à partir de $K_{6}$ par des transformations étoile-triangle. 


\section{ProBlème}

La question se pose de savoir s'il y a d'autres graphes 5-critiques. Un résultat de Robertson, SEymour et Thomas [RST1] et [RST2] affirme que les sept graphes obtenus à partir de $K_{6}$ par des transformations étoiletriangle sont les seuls graphes critiques pour le problème du plongement plat dans $\mathbb{R}^{3}$ (un plongement $\Gamma \subset \mathbb{R}^{3}$ est plat si tout cycle simple de $\Gamma$ est bord d'un disque ouvert contenu dans $\mathbb{R}^{3} \backslash \Gamma$ ). La question ci-dessus se reformule donc comme suit : si $\Gamma$ admet un plongement plat dans $\mathbb{R}^{3}$, a-t-on nécessairement $\mu(\Gamma) \leq 4$ ?

Il serait en particulier utile de calculer $\mu$ pour un graphe qui admet un plongement plat dans $\mathbb{R}^{3}$ sans qu'il soit possible de le rendre planaire par suppression d'un sommet. Le graphe $\Gamma$ obtenu à partir du graphe biparti complet $K_{5,5}$ en enlevant les arêtes $\left(1,1^{\prime}\right),\left(2,2^{\prime}\right), \ldots,\left(5,5^{\prime}\right)$ (avec des notations évidentes) a cette propriété (exemple cité dans [RST1]).

\section{REMARques}

Le ThÉORÈme 2 implique en particulier que supprimer des sommets de degré 1 ne change pas $\mu$ dès que $\mu$ vaut au moins 3 . De même, contracter une arête incidente en un sommet de degré 2 ne change pas $\mu$ dès que $\mu$ vaut au moins 4 . Ceci montre en particulier que l'invariant $\mu$ est un invariant topologique pour les graphes non-planaires.

En ce qui concerne la recherche des graphes $n$-critiques, les THÉORÈMES 2 et 3 montrent que l'on peut restreindre la recherche des graphes $n$ critiques $(n \geq 5)$ aux graphes finis connexes simples dont tous les sommets sont de degré $\geq 4$.

On dira d'une transformation $\Delta Y$ d'un graphe planaire $\Gamma$ qu'elle est planaire si au plus une seule composante connexe de $\Gamma \backslash \Delta$ (le graphe obtenu à partir de $\Gamma$ en supprimant les trois sommets de $Y$ ainsi que toutes les arêtes incidentes en un de ces sommets) est adjacente au trois sommets de $Y$.

Les ThÉORÈmEs 1 et 2 permettent de redémontrer par une preuve combinatoire le théorème 3.2 de [CV1] qui dit que l'invariant $\mu$ d'un graphe planaire vaut au plus 3 (l'invariant $\mu$ d'un graphe non planaire est lui supérieur ou égal à 4 : cela résulte du critère de Kuratowski, du fait que l'invariant $\mu$ des 2 graphes de Kuratowski est 4 et du fait que $\mu$ est croissante pour la relation de mineur (voir [CV1] ou [CV3]). Pour ceci on utilise la description suivante des graphes planaires.

Proposition 1. - Tout graphe planaire connexe se réduit à un point par une suite finie de transformations:

(i) effacer une arête incidente en un sommet de degré 1 (et supprimer le sommet isolé ainsi créé); 
(i') effacer une boucle;

(ii) remplacer deux arêtes incidentes en un sommet commun de degré 2 par une unique arête;

(ii') remplacer une arête double par une arête simple;

(iii) les transformations $Y \Delta$ régulières;

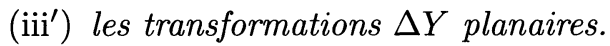

(Rappelons qu'une transformation Y $\mathrm{Y}$ est régulière si le mineur obtenu en effaçant les quatre sommets de $Y$ et toutes les arêtes incidentes en au moins un de ces sommets reste connexe. Rappellons aussi qu'une transformation $\Delta Y$ est planaire si elle est réalisable sur des graphes planaires.)

Notons que ces transformations préservent la planarité.

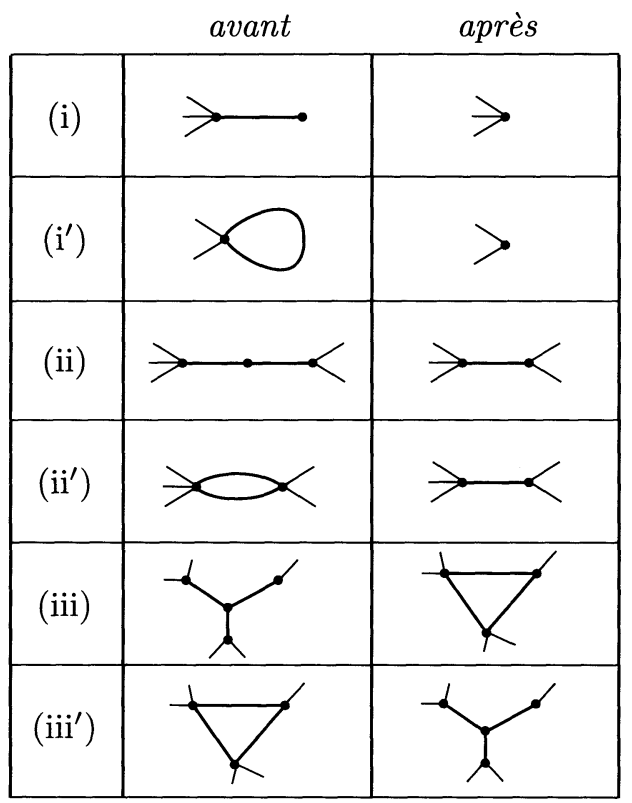

Figure 3. Les transformations de la proposition 1.

Corollaire 1 (théorème 3.2 dans [CV1]). - Si $\Gamma$ est un graphe planaire on $a \mu(\Gamma) \leq 3$.

Preuve. - Soit $\Gamma$ un graphe planaire. Si $\mu(\Gamma) \geq 4$, les opérations décrites dans la Proposition 1 préservent $\mu$ par les ThÉorèmes 1 et 2 . On arrive donc à une contradiction. 
Une autre preuve combinatoire du Corollaire 1 est donnée dans [vHLS].

Nous remercions François JAEger et Robin Thomas pour des discussions, questions et informations, ainsi que Laurent GuILlopé et Pierre DE LA HARPE pour leurs remarques sur une première version de ce texte.

\section{Rappels}

Voici quelques rappels de [CV1]. Soit $\Gamma$ un graphe fini, connexe et simple (c'est-à-dire sans boucle et sans arête multiple). On note $E$ l'ensemble de ses arêtes et $V$ celui de ses sommets. On désigne par $\mathcal{O}_{\Gamma}$ le cône des endomorphismes symétriques $A$ de $\mathbb{R}^{V}$ tels que les coefficients de $A$ vérifient :

$$
\left\{\begin{array}{l}
a_{i, j}<0 \text { si }(i, j) \in E, \\
a_{i, j}=0 \text { si } i \neq j \text { et }(i, j) \notin E .
\end{array}\right.
$$

Les valeurs propres de $A$ répétées avec leurs multiplicités vérifient :

$$
\lambda_{1}<\lambda_{2} \leq \lambda_{3} \leq \cdots \leq \lambda_{\sharp V}
$$

Pour tout entier $n \geq 1$, on désigne par $W_{\lambda, n}$ la sous-variété algébrique de l'espace des endomorphismes symétriques de $\mathbb{R}^{V}$ contenant les matrices $A$ admettant $\lambda$ comme valeur propre avec multiplicité au moins $n$.

Soient $i$ et $n$ deux entiers naturels tels que $2 \leq i \leq i+n-1 \leq \sharp V$, soit $A \in \mathcal{O}_{\Gamma}$ et soit $\lambda_{i}$ la $i$-ième valeur propre de $A$. On dit que $\lambda_{i}$ est stable $(\mathrm{SAH})$ de multiplicité $n$ si les valeurs propres de $A$ vérifient

$$
\lambda_{1} \leq \lambda_{2} \leq \cdots \leq \lambda_{i-1}<\lambda_{i}=\cdots=\lambda_{i+n-1}<\lambda_{i+n} \leq \cdots \leq \lambda_{\sharp V}
$$

et si de plus $\mathcal{O}_{\Gamma}$ et $W_{\lambda_{i}, n}$ se coupent transversalement en $A$. Un critère algébrique pour cette transversalité est que, si $F\left(\lambda_{i}\right)=\operatorname{Ker}\left(A-\lambda_{i}\right)$, on ait:

Les formes quadratiques $x_{i} x_{j},(i, j) \in E$ et $x_{i}^{2}, i \in V$ restreintes à $F\left(\lambda_{i}\right)$ engendrent l'espace de toutes les formes quadratiques sur $F\left(\lambda_{i}\right)$.

On définit $\mu(\Gamma)$ comme le plus grand entier $n$ tel qu'il existe une matrice $A \in \mathcal{O}_{\Gamma}$ pour laquelle la deuxième valeur propre $\lambda_{2}$ est stable de multiplicité $n$.

On peut étendre la définition de l'invariant $\mu$ à des graphes finis quelconques. La stabilité de $F\left(\lambda_{2}\right)$ implique alors que le support de $F\left(\lambda_{2}\right)$ est contenu dans une seule composante connexe.

On dit qu'un graphe $\widetilde{\Gamma}$ est mineur d'un graphe $\Gamma$ si on peut passer de $\Gamma$ à $\widetilde{\Gamma}$ par une suite d'opérations de suppressions et de contractions d'arêtes.

TOME $123-1995-\mathrm{N}^{\circ} 4$ 
L'invariant $\mu$ possède la propriété intéressante d'être décroissant pour les mineurs : si $\widetilde{\Gamma}$ est mineur de $\Gamma$, on a :

$$
\mu(\widetilde{\Gamma}) \leq \mu(\Gamma) .
$$

Un graphe fini connexe simple est dit $n$-critique pour l'invariant $\mu$ si $\mu(\Gamma)=n$ et si $\mu(\widetilde{\Gamma})<n$ pour tout mineur strict $\widetilde{\Gamma}$ de $\Gamma$. Il est intéressant de connaître la liste des graphes simples connexes qui sont $n$-critiques.

La liste complète de ces graphes est connue pour $n \leq 4$ [CV1] :

- le graphe complet sur $n$ sommets est $(n-1)$-critique $(n \geq 1)$;

- l'étoile $K_{3,1}$ (notée $Y$ ci-dessus) est 2-critique;

- le graphe biparti complet $K_{3,2}$ est 3 -critique;

- le graphe de Kuratowski $K_{3,3}$ est 4-critique.

\section{Preuve du théorème 1}

On considère un graphe $\Gamma$ d'ensemble de sommets $V=\{0,1, . ., N\}$. On suppose que $\{0,1,2,3\}$ sont les sommets d'une étoile de centre 0 . Notons $\Gamma^{\prime}$ le graphe obtenu à partir de $\Gamma$ par une transformation $Y \Delta$ par rapport à ces quatre sommets. Quitte à remplaçer $\Gamma$ par un mineur on peut supposer que les sommets 1,2 et 3 de $\Gamma$ ne sont pas reliés par des arêtes. Il faut montrer qu'on a $\mu(\Gamma) \geq \mu\left(\Gamma^{\prime}\right)$.

On donne ici une preuve du ThÉORÈme 1 basée sur les idées de [CV2].

Une difficulté essentielle dans la preuve de ce théorème est que l'ensemble des sommets $V=\{0,1, \ldots, N\}$ de $\Gamma$ n'est pas le même que l'ensemble des sommets du transformé $\Gamma^{\prime}$ par $Y \Delta$ de $\Gamma$ qui est $\{1,2, \ldots, N\}$. On doit donc traiter de limites de formes quadratiques dans une situation où la limite est définie sur un sous-espace. Dans [CV2], on a introduit dans ce but une compactification de l'espace des formes quadratiques $Q_{X}$ sur un espace vectoriel réel $X$ de dimension fini. Cette compactification est donnée par la grassmannienne lagrangienne $\mathcal{L}_{X}$ de $X \oplus X^{*}$ muni de la structure symplectique canonique. On montre que $\mathcal{L}_{X}$ s'identifie naturellement à l'ensemble des couples $(q, Y)$ d'une forme quadratique $q$ définie sur le sous-espace $Y$ de $X$ grâce à l'application

$$
(q, X) \longmapsto L
$$

avec

$$
L=\left\{(y, \xi) \mid y \in Y, \xi(z)=A_{q}(z)(y) \text { pour tout } z \in Y\right\}
$$

où $A_{q}: Y \longrightarrow Y^{*}$ est l'application linéaire symétrique associée à $q$. 
On peut donc maintenant considérer $O_{\Gamma}$ ainsi que $O_{\Gamma^{\prime}}$ comme des sousvariétés de $\mathcal{L}=\mathcal{L}_{\mathbb{R}^{V}}$. Soient $\mu=\mu\left(\Gamma^{\prime}\right)$ et

$$
W_{\mu}=\left\{L \in \mathcal{L} \mid \operatorname{dim}\left(L \cap\left(\mathbb{R}^{V} \oplus 0\right)\right)=\mu\right\}
$$

Alors $W_{\mu}$ est une sous-variété de $\mathcal{L}$ de codimension $\frac{1}{2} \mu(\mu+1)$ et on a :

LeMme 1. - Il existe $A_{0} \in O_{\Gamma^{\prime}}$ tel que $O_{\Gamma^{\prime}}$ coupe $W_{\mu}$ transversalement en $A_{0}$.

Preuve. - On choisit un $A$ qui réalise $\mu\left(\Gamma^{\prime}\right)$ et le modifie en $A_{0}$ par un multiple de Id pour que $\lambda_{2}\left(A_{0}\right)=0$. Alors $O_{\Gamma^{\prime}}$ coupe l'intersection de $W_{\mu}$ avec les formes quadratiques sur $\mathbb{R}^{V^{\prime}}$ transversalement en $A_{0}$. Mais il est facile de voir que $W_{\mu}$ et la variété des formes de domaine $\mathbb{R}^{V^{\prime}}$ sont transverses en $A_{0}$. Ces formes induisent effet une forme quadratique arbitraire sur $\operatorname{Ker}\left(A_{0}\right) \subset \mathbb{R}^{V^{\prime}}$.

Définissons, pour $\epsilon>0$, une sous-variété $Z_{\epsilon}$ de $O_{\Gamma}$ et donc de $\mathcal{L}$ par

$$
Z_{\epsilon}=\left\{q_{\epsilon}(x)=\sum_{i=1}^{3} c_{i}\left(x_{i}-\frac{x_{0}}{\epsilon}\right)^{2}+Q\left(x_{1}, \ldots, x_{N}\right)\right\}
$$

avec $c_{1}, c_{2}, c_{3}>0$ et $Q \in O_{\Gamma_{0}}$ où $\Gamma_{0}$ est le graphe obtenu en privant $\Gamma$ du sommet 0 et des 3 arêtes qui en sont issues. On remarque que $Z_{\epsilon}$ a la même dimension que $O_{\Gamma^{\prime}}$.

La clé de la preuve est donnée par le lemme suivant.

Lemme 2. - Lorsque $\epsilon \rightarrow 0^{+}$, la sous-variété $Z_{\epsilon}$ converge au sens $C^{\infty}$ sur tout compact vers la sous-variété $O_{\Gamma^{\prime}}$.

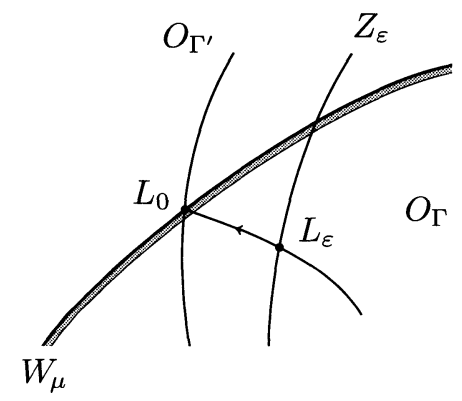

Figure 4.

TOME $123-1995-\mathrm{N}^{\circ} 4$ 
Preuve. - Il s'agit de montrer que les espaces lagrangiens $L_{\epsilon}$ associés à $q_{\epsilon}$ convergent au sens $C^{\infty}$ (par rapport à $(c, Q)$ ) vers l'espace $L_{0} \in O_{\Gamma^{\prime}}$ où $L_{0}$ est associé à la forme quadratique

$$
q_{0}(x)=\sum_{i=1}^{3} \frac{c_{i} c_{i+1}}{c_{1}+c_{2}+c_{3}}\left(x_{i}-x_{i+1}\right)^{2}+Q\left(x^{\prime}\right)
$$

où le paramètre $i$ est pris modulo 3 et $x=\left(x_{0}, x^{\prime}\right)$. (L'application

$$
\left(c_{1}, c_{2}, c_{3}\right) \longmapsto(a, b, c)=\left(\frac{c_{2} c_{3}}{c_{1}+c_{2}+c_{3}}, \frac{c_{1} c_{3}}{c_{1}+c_{2}+c_{3}}, \frac{c_{1} c_{2}}{c_{1}+c_{2}+c_{3}}\right)
$$

est un difféomorphisme de $\left(\mathbb{R}_{>0}\right)^{3}$ d'inverse

$$
(a, b, c) \longmapsto\left(\frac{b c+a c+a b}{a}, \frac{b c+a c+a b}{b}, \frac{b c+a c+a b}{c}\right) .
$$

Cette application est bien connue en électricité, $c f$. par exemple [Bo, p. 30-31].

Explicitons les équations de $L_{\epsilon}$, en notant :

$$
\ell_{i}\left(x^{\prime}\right)=\frac{1}{2} \frac{\partial Q}{\partial x_{i}}
$$

Par abus de notation, les autres occurences de $x_{0}, \ldots, x_{N}$ désigneront les éléments de la base évidente de $\mathbb{R}^{V}$ et $\xi_{0}, \ldots, \xi_{N}$ désignera la base duale :

$$
\left\{\begin{aligned}
\xi_{0} & =-\frac{1}{\epsilon} \sum c_{j}\left(x_{j}-\frac{x_{0}}{\epsilon}\right) ; & & \\
\xi_{i} & =c_{i}\left(x_{i}-\frac{x_{0}}{\epsilon}\right)+\ell_{i}\left(x^{\prime}\right) & & i=1,2,3 ; \\
\xi_{i} & =\ell_{i}\left(x^{\prime}\right) & & i>3 .
\end{aligned}\right.
$$

On peut réécrire ces $N+1$ équations sous la forme équivalente :

$$
\begin{cases}\left(\sum c_{j}\right) x_{0}=\epsilon \sum c_{j} x_{j}+\epsilon^{2} \xi_{0} ; & \\ \sum_{i=1}^{3}\left(\xi_{i}-\ell_{i}\left(x^{\prime}\right)\right)=-\epsilon \xi_{0} ; & \\ c_{i+1}\left(\xi_{i}-\ell_{i}\left(x^{\prime}\right)\right)-c_{i}\left(\xi_{i+1}-\ell_{i+1}\left(x^{\prime}\right)\right) & \\ -c_{i} c_{i+1}\left(x_{i}-x_{i+1}\right)=0 & i=1,2,3 ; \\ \xi_{i}-\ell_{i}\left(x^{\prime}\right)=0 & i>3 .\end{cases}
$$


En posant $\eta_{i}=\xi_{i}-\ell_{i}\left(x^{\prime}\right)$ pour $i=1,2,3$, on obtient en prenant la deuxième équation et les deux suivantes un système de trois équations que l'on peut résoudre explicitement en $\eta_{i}$.

On a ainsi les équations de $L_{\epsilon}$ sous la forme :

$$
\left\{\begin{array}{rr}
x_{0}=\frac{\epsilon}{c_{1}+c_{2}+c_{3}}\left(c_{1} x_{1}+c_{2} x_{2}+c_{3} x_{3}\right)+\frac{\epsilon^{2}}{c_{1}+c_{2}+c_{3}} \xi_{0} & \\
\xi_{i}=\frac{c_{i} c_{i-1}}{c_{1}+c_{2}+c_{3}}\left(x_{i}-x_{i-1}\right)+\frac{c_{i} c_{i+1}}{c_{1}+c_{2}+c_{3}}\left(x_{i}-x_{i+1}\right) & \\
+\ell_{i}\left(x^{\prime}\right)+\epsilon c_{i} \xi_{0} & i=1,2,3 ; \\
\xi_{i}=\ell_{i}\left(x^{\prime}\right) & i>3 .
\end{array}\right.
$$

Cela montre la dépendance analytique de $L_{\epsilon}$ par rapport à $\left(\epsilon, c_{1}, c_{2}, c_{3}, Q\right)$ au voisinage de $\epsilon=0$ et conclut la preuve.

Le THÉoRÈme 1 en résulte facilement, car alors $W_{\mu}$ coupe $Z_{\epsilon}$ transversalement en $A_{\epsilon}$ pour $\epsilon>0$ assez petit.

\section{Preuve du théorème 2}

On a besoin du résultat suivant.

LEMME. - On considère une transformation $* S$ d'un graphe $\Gamma$ par rapport à un sommet de degré $k$. On suppose que les hypothèses $d u$ théorème 2 sont vérifiées. Notons $0,1, \ldots, k$ les $(k+1)$ sommets impliqués dans la transformation $* S$ où 0 est le sommet central de $*$. Soit $A \in \mathcal{O}_{\Gamma}$ un endomorphisme symétrique qui réalise $\mu(\Gamma)$ et soit $\lambda_{2}$ la deuxième valeur propre de multiplicité $n=\mu(\Gamma)$ de A. Supposons que

$$
A x_{0}=V_{0} x_{0}-\sum_{i=1}^{k} c_{i} x_{i} \quad \text { ò̀ } c_{i}>0
$$

Alors $\lambda_{2}<V_{0}$.

Preuve. - Montrons le lemme d'abord dans le cas où $n=\mu(\Gamma) \geq k+1$ et où la transformation $* S$ considérée est régulière.

Considérons l'espace des fonctions de $V$ dans $\mathbb{R}$ comme espace euclidien muni de la base orthonormée $\left\{x_{0}, \ldots, x_{N}\right\}$ donnée par les fonctions caractéristiques des sommets de $\Gamma$. Soit $F\left(\lambda_{2}\right)=\operatorname{Ker}\left(A-\lambda_{2}\right)$ l'espace propre associé à $\lambda_{2}$. Il existe

$$
\varphi=\sum_{i=0}^{N} \alpha_{i} x_{i} \in F\left(\lambda_{2}\right)
$$

TOME $123-1995-\mathrm{N}^{\circ} 4$ 
tel que $\alpha_{0}=\alpha_{1}=\ldots=\alpha_{k}=0$ et $\varphi$ est de norme 1. En effet, si $V_{0} \neq \lambda_{2}$, la nullité de $\alpha_{1}, \alpha_{2}, \ldots, \alpha_{k}$ implique la nullité de $\alpha_{0}$. Dans le cas contraire la nullité de $\alpha_{1}, \alpha_{2}, \ldots, \alpha_{k-1}$ et $\alpha_{0}$ implique la nullité de $\alpha_{k}$. La plus petite valeur propre $\lambda_{1}$ de $A$ est réalisé par un vecteur de Perron-Frobenius noté $\pi$. On a donc :

$$
\pi=\sum_{i=0}^{N} \beta_{i} x_{i} \quad \text { avec } \beta_{i}>0 \text { pour } i=0, \ldots, N .
$$

Comme les vecteurs $\varphi$ et $\pi$ sont des vecteurs propres associés à des valeurs propres distinctes de $A$, ils sont orthogonaux. Les coefficients $\alpha_{i}$ de $\varphi$ n'ont donc pas tous le même signe.

Soit $\widetilde{\Gamma}$ le graphe obtenu en supprimant les sommets $0,1,2, \ldots, k$ (ainsi que les arêtes incidentes en un de ces sommets) de $\Gamma$. Considérons le problème de Dirichlet dans $\widetilde{\Gamma}$ avec bord $\{0,1,2, \ldots, k\}$. Il consiste à étudier l'endomorphisme symétrique $\widetilde{A}=P A P \in \mathcal{O}_{\widetilde{\Gamma}}$ où $P$ est la projection

$$
P\left(\xi_{0}, \xi_{1}, \ldots, \xi_{k}, \xi_{k+1}, \ldots, \xi_{N}\right)=\left(0,0, \ldots, 0, \xi_{k+1}, \ldots, \xi_{N}\right)
$$

La fonction

$$
\widetilde{\varphi}=\sum_{i=k+1}^{N} \alpha_{i} x_{i}
$$

est un vecteur propre de la restriction de $\widetilde{A}$ à $P \mathbb{R}^{N+1}$. Comme la transformation $* S$ considérée est régulière, la restriction de l'endomorphisme $\widetilde{A}$ à $P \mathbb{R}^{N+1}$ possède un vecteur de Perron-Frobenius $\widetilde{\sim}$. Notons $\psi$ le vecteur de norme 1 de $V$ dont la restriction à $\widetilde{\Gamma}$ est $\widetilde{\psi}$ et qui s'annulle sur $(0,1, \ldots, k)$. Le vecteur $\psi$ est linéairement indépendant de $\varphi$ car les coefficients de $\varphi$ ne sont pas de signe constant. Pour la valeur propre $\tilde{\lambda}_{1}$ de $\widetilde{\psi}$ on a donc $\tilde{\lambda}_{1}<\lambda_{2}$. Ceci montre qu'il existe $\alpha, \beta \in \mathbb{R} \backslash\{0\}$ tel que $\alpha \psi+\beta x_{0}$ est un vecteur de norme 1 qui est orthogonal à $\pi$. Ceci implique qu'on a :

$$
\lambda_{2} \leq\left\langle A\left(\alpha \psi+\beta x_{0}\right) \mid\left(\alpha \psi+\beta x_{0}\right)\right\rangle=\alpha^{2} \tilde{\lambda}_{1}+\beta^{2} V_{0}
$$

Les conditions $\alpha^{2}+\beta^{2}=1$ et $\tilde{\lambda}_{1}<\lambda_{2}$ impliquent bien $\lambda_{2}<V_{0}$ ce qui démontre le lemme dans le cas d'une transformation $* S$ régulière.

Montrons maintenant le lemme dans le cas où $\mu(\Gamma) \geq k+2$ et où la transformation $* S$ n'est pas régulière. On peut donc trouver deux vecteurs linéairement indépendants $\varphi$ et $\varphi^{\prime}$ de $F\left(\lambda_{2}\right)$ qui s'annulent sur les sommets $0,1, \ldots, k$. Définissons comme plus haut le graphe

$$
\widetilde{\Gamma}=\Gamma \backslash\{0,1, \ldots, k\}
$$


S'il existe une composante connexe $\widetilde{\Gamma}_{c}$ de $\widetilde{\Gamma}$ telle que les restrictions de $\varphi$ et $\varphi^{\prime}$ à $\widetilde{\Gamma}_{c}$ sont linéairement indépendantes, on a fini en prenant comme vecteur $\psi$ le vecteur de Perron-Frobenius associé à la composante connexe $\widetilde{\Gamma}_{c}$ et en poursuivant comme ci-dessus, avec une combinaison linéaire de $\varphi$ et $\varphi^{\prime}$ non proportionnelle à $\psi$.

On peut donc supposer que les restrictions de $\varphi$ et $\varphi^{\prime}$ à toutes les composantes connexes de $\widetilde{\Gamma}$ sont linéairement dépendantes. L'espace des formes quadratiques sur $\mathbb{R} \varphi+\mathbb{R} \varphi^{\prime}$ est de dimension 3. La stabilité de $F\left(\lambda_{2}\right)$ montre donc qu'il existe au moins trois composantes connexes distinctes de $\widetilde{\Gamma}$ sur lesquelles $\varphi$ et $\varphi^{\prime}$ ne s'annullent pas simultanément. Choisissons trois telles composantes $\widetilde{\Gamma}_{1}, \widetilde{\Gamma}_{2}, \widetilde{\Gamma}_{3} \subset \widetilde{\Gamma}$ et choisissons trois vecteurs $\varphi_{i}$ (pour $i=1,2,3$ ) de norme 1 , identiquement zéro en dehors de $\widetilde{\Gamma}_{i}$ et qui sont égaux à la restriction d'un multiple de $\varphi$ ou de $\varphi^{\prime} \operatorname{sur} \widetilde{\Gamma}_{i}$.

Pour $i=1,2,3$ choisissons maintenant $\alpha_{i}, \beta_{i} \in \mathbb{R}$ tel que les vecteurs $\alpha_{i} \varphi_{i}+\beta_{i} x_{0}$ sont de norme 1 et orthogonaux au vecteur de PerronFrobenius $\pi$. En raisonnant comme plus haut on voit qu'on a

$$
\lambda_{2} \leq \max \left(\lambda_{2}, V_{0}\right)
$$

avec égalité si et seulement si les trois vecteurs $\alpha_{i} \varphi_{i}+\beta_{i} x_{0}$ sont vecteurs propres de valeur propre $\lambda_{2}$ ce qui implique $V_{0}=\lambda_{2}$. Supposons d'abord que $\beta_{1}$ est non nul. Dans ce cas considérons les deux vecteurs propres (non-nuls)

$$
\left(\beta_{1} \alpha_{2} \varphi_{2}-\beta_{2} \alpha_{1} \varphi_{1}\right) \text { et } \alpha_{3} \varphi_{3}+\beta_{3} x_{0} .
$$

Ils appartiennent tous les deux à $F\left(\lambda_{2}\right)$ et ils ont des supports disjoints qui ne sont reliés par aucune arête de $\Gamma$. L'espace $F\left(\lambda_{2}\right)$ ne vérifie donc pas le critère pour la stabilité. Dans le cas où les $\beta_{i}$ sont tous nuls (et où les $\varphi_{i}$ sont des vecteurs propres) on applique l'argument précédent aux deux vecteurs $\varphi_{1}$ et $\varphi_{2}$.

En reprenant les notations du Lemme, on peut supposer $\lambda_{2}=0, F=$ $F\left(\lambda_{2}\right)=\operatorname{Ker}(A)$, et donc $V_{0}>0$. Soit :

$$
L\left(x_{1}, \ldots, x_{k}\right)=\frac{1}{V_{0}} \sum_{i=1, \ldots, k} c_{i} x_{i}
$$

on a $x_{0}=L\left(x_{1}, \ldots, x_{k}\right)$ pour $\left(x_{0}, x_{1}, \ldots, x_{N}\right) \in F$. Soit alors $\mathcal{E}$ le sousespace de $\mathbb{R}^{N+1}$ défini par cette équation, c'est-à-dire :

$$
\mathcal{E}=A^{-1}\left(0 \oplus \mathbb{R}^{N}\right)=\left(L\left(x_{1}, \ldots, x_{k}\right), x_{1}, x_{2}, \ldots, x_{N}\right) .
$$

Définissons $A^{\prime}: \mathbb{R}^{N} \longrightarrow \mathbb{R}^{N}$ par

$$
A^{\prime}\left(x_{1}, x_{2}, \ldots, x_{N}\right)=\pi\left(A\left(L\left(x_{1}, \ldots, x_{k}\right), x_{1}, \ldots, x_{N}\right)\right),
$$

TOME $123-1995-\mathrm{N}^{\circ} 4$ 
où $\pi: \mathbb{R}^{N+1} \longrightarrow \mathbb{R}^{N}$ est la projection définie par :

$$
\pi\left(x_{0}, x_{1}, \ldots, x_{N}\right)=\left(x_{1}, \ldots, x_{N}\right) .
$$

Il est facile de vérifier que $A^{\prime}$ est symétrique, appartient à $O_{\Gamma^{\prime}}$ et que le noyau de $A^{\prime}$ est la projection par $\pi$ du noyau $F$ de $A$.

Pour $x=\left(x_{0}, x_{1}, \ldots, x_{N}\right)$ et $x^{\prime}=\left(x_{1}^{\prime}, \ldots, x_{N}^{\prime}\right)$ notons

$$
q(x)=\langle x \mid A x\rangle \quad \text { et } \quad q^{\prime}\left(x^{\prime}\right)=\left\langle x^{\prime} \mid \pi\left(A\left(L\left(x_{1}^{\prime}, \ldots, x_{3}^{\prime}\right), x_{1}^{\prime}, \ldots, x_{N}^{\prime}\right)\right)\right\rangle
$$

les formes quadratiques associées à $A$ et $A^{\prime}$.

Par le principe du minimax, il est clair, en identifiant $q^{\prime}$ à la restriction de $q$ à $\mathcal{E}$, que la signature de $q^{\prime}$ est $(N-n-1,1, n)\left(\operatorname{car} \pi(F)=\operatorname{Ker}\left(q^{\prime}\right)\right)$. On en déduit que 0 est la seconde valeur propre de $A^{\prime}$ et que sa multiplicité est $n$.

La vérification que 0 est stable $(\mathrm{SAH})$ de multiplicité $n$ pour $A^{\prime}$ à partir de la même hypothèse pour $A$ se fait sans difficulté en remarquant que les formes quadratiques $x_{i}^{2}, x_{i} x_{j}$, pour $1 \leq i, j \leq k$ induisent sur $F$ un sous-espace de formes quadratiques au moins aussi grand que les formes $x_{i}^{2}, x_{0} x_{i}, x_{0}^{2}$ pour $1 \leq i \leq k$.

\section{Preuve du théorème 3}

Soit $\Gamma$ et $\Gamma^{\prime}$ deux graphes finis reliés par une transformation étoiletriangle. Si $\mu(\Gamma) \geq 5$ ou $\mu\left(\Gamma^{\prime}\right) \geq 5$ les ThÉORÈmES 1 et 2 impliquent qu'on a $\mu(\Gamma)=\mu\left(\Gamma^{\prime}\right)$.

Supposons qu'un des deux graphes soit $\mu$-critique. La criticité de l'autre graphe par rapport aux arêtes non concernées par la transformation étoiletriangle est claire. Pour les trois arêtes concernées, il suffit de remarquer que les deux graphes admettent, après suppression éventuelle d'un sommet de degré 2 , les mêmes mineurs immédiats. Or supprimer un sommet de degré 2 ne change pas $\mu$ pourvu qu'il soit au moins égal à 4 .

On en déduit que tous les graphes obtenus par transformations étoiletriangle à partir du graphe complet à $N$ sommets $K_{N}$ avec $N \geq 6$ sont $(N-1)$-critiques pour l'invariant $\mu$.

\section{Preuve de la proposition 1}

La Proposition 1 est vraie lorsqu'on remplaçe (iii) par :

(iiif) une transformation $Y \Delta$.

Ceci découle d'un résultat d'Epifanov dont nous enonçons ici une version faible (voir [Gr, chap. 13] ou [E]). 
LEMME. - Tout graphe planaire connexe fini se réduit à un point par une suite finie de transformations :

(i) effacer une arête incidente en un sommet de degré 1 (et supprimer le sommet isolé ainsi crée);

(i') effacer une boucle;

(ii) remplacer deux arêtes incidentes en un sommet commun de degré 2 par une unique arête;

(ii') remplacer une arête double par une arête simple;

(iiif) $Y \Delta$;

(iii') $\Delta Y$ planaire.

Preuve du lemme. - La preuve est par récurrence sur le nombre $e$ d'arêtes de $\Gamma$.

Soit $\Gamma$ un graphe plongé dans le plan $E$. Chaque composante connexe de $E \backslash \Gamma$ (y compris la composante non-bornée) est une face de $(\Gamma, E)$ dont le bord est un cycle d'arêtes de $\Gamma$. Le graphe médial $M(\Gamma)$ de $\Gamma$ est un graphe planaire dont les sommets sont les milieux des arêtes de $\Gamma$ et dont les arêtes sont définies comme suit : deux sommets $x_{1}, x_{2}$ de $M(\Gamma)$ correspondants à des arêtes $e_{1}, e_{2}$ de $\Gamma$ sont liées par une arête de $M(\Gamma)$ chaque fois que $e_{1}, e_{2}$ sont des arêtes consécutives d'une même face de $G$. Voici deux exemples sur la figure 5 .
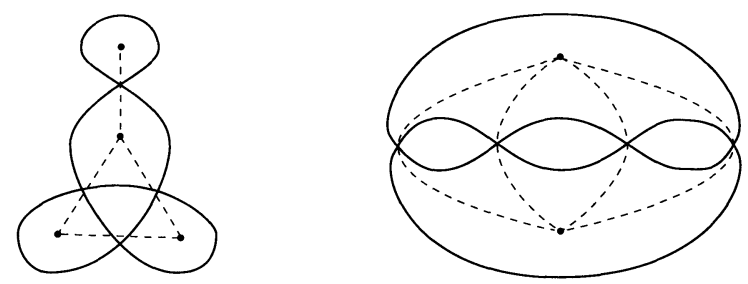

Figure 5. Deux exemples de graphes médiaux.

Un graphe médial (convenablement dessiné) est une immersion d'une union finie de cercles dans $\mathbb{R}^{2}$.

Soit $M(\Gamma)$ un graphe médial. Une lentille $L$ de $M(\Gamma)$ est un ouvert borné connexe simplement connexe de $\mathbb{R}^{2}$ tel qu'il existe deux difféomorphismes

$$
\ell_{1}, \ell_{2}:[0,1] \longrightarrow \partial L \subset M(\Gamma)
$$

avec :

$$
\begin{cases}\ell_{1}(0)=\ell_{2}(0), & \ell_{1}(1)=\ell_{2}(1) \\ \ell_{i}(s) \neq \ell_{i}(t) & \text { pour } i=1,2 \text { et } 0 \leq s<t \leq 1 \\ \ell_{1}(s) \neq \ell_{2}(t) & \text { pour } 0<s, t<1\end{cases}
$$

TOME $123-1995-\mathrm{N}^{\circ} 4$ 


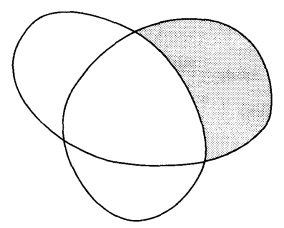

Figure 6. Une lentille dans un graphe médial.

Une lentille $L$ est vide si $M(\Gamma) \subset \mathbb{R}^{2} \backslash L$. Une lentille vide dans $M(\Gamma)$ provient d'un graphe $\Gamma$ qui peut se réduire par une opération (ii) ou (ii'). D'autre part, une lentille non vide minimale (ie ne contenant pas de souslentilles) de $M(\Gamma)$ peut être vidée par des opérations qui correspondent à des transformations de type (ii), (ii'), $Y \Delta$ et $\Delta Y$ planaires sur le graphe $\Gamma$.

Montrons donc la proposition par récurrence sur le nombre $e$ d'arêtes de $\Gamma$.

- Si $e=0$ il n'y a rien à faire.

- Supposons la proposition démontrée pour tout graphe connexe planaire ayant moins que $e$ arêtes. Soit $\Gamma$ un graphe avec $e$ arêtes. Considérons le graphe médial $M(\Gamma)$ associé à $\Gamma$. Si $M(\Gamma)$ ne contient aucune lentille, le graphe $\Gamma$ peut se réduire à un point uniquement par des opérations du type (i) et $\left(\mathrm{i}^{\prime}\right)$. La proposition est donc vraie dans ce cas. Sinon soit $A$ une lentille de $M(\Gamma)$. On peut vider $A$ par des transformations qui correspondent à des transformations (ii), (ii'), $Y \Delta$ et $\Delta Y$ planaires sur $\Gamma$ (ce qui n'augmente pas le nombre d'arêtes de $\Gamma$ ). On arrive finalement à un graphe qui se réduit par une opération (ii) ou (ii') à un graphe $\Gamma^{\prime}$. Comme le passage de $\Gamma$ à $\Gamma^{\prime}$ fait intervenir au moins une transformation de type (ii) ou (ii'), le graphe $\Gamma^{\prime}$ a au moins une arête de moins que $\Gamma$. L'hypothèse de récurrence s'applique donc à $\Gamma^{\prime}$.

Preuve de la proposition 1.

Elle est analogue à la preuve du lemme précédent. Appelons transformations admissibles les transformations de la Proposition 1. Considérons une lentille du graphe médial $M(\Gamma)$. Si on peut la vider par des transformations admissibles on a terminé par récurrence sur le nombre d'arêtes de $\Gamma$.

On peut donc supposer qu'on utilise une transformation $Y \Delta$ nonrégulière pour vider la lentille. Considérons le sous-graphe $Y$ de $\Gamma$ pour lequel la transformation $Y \Delta$ considérée n'est pas régulière. Notons $C_{1}, \ldots, C_{k}$ (avec $k \geq 2$ car la transformation considérée n'est pas régulière) les composantes connexes de $\Gamma \backslash Y$. Si deux des composantes $C_{1}, . ., C_{k}$ étaient reliées par des arêtes aux trois sommets extérieurs de $Y$, le graphe $\Gamma$ admettrait le graphe de Kuratowski $K_{3,3}$ comme mi- 
neur ce qui contredirait sa planarité. Il existe donc au plus un graphe parmi $C_{1}, . ., C_{k}$ qui est relié au trois sommets extérieurs de $Y$. Associons à chaque composante connexe $C_{i}$ un graphe $\widetilde{C}_{i}$ construit comme suit :

- D'abord on adjoint à $C_{i}$ les trois sommets extérieurs de $Y$ et toutes les arêtes reliant un de ces trois sommets à $C_{i}$. Finalement on enlève les sommets isolés ainsi crées ( $\mathrm{s}^{\prime} \mathrm{il}$ y en a).

- Si $k \geq 3$, choisissons parmi les graphes $\widetilde{C}_{i}$ contenant au plus deux sommets extérieurs de $Y$ un avec le nombre minimal d'arêtes et appelonsle $C$.

- Si $k=2$, appelons $C$ le graphe $\widetilde{C}_{i}$ avec le moins d'arêtes.

Soit $j$ le nombre de sommets extérieurs de $Y$ contenus dans $C$. On a donc $j=1,2$ ou 3. Interprétons $C$ comme un graphe dont les $j$ sommets provenant de $Y$ sont marqués. On peut associer à $C$ un graphe médial $M(C)$ avec $2 j$ bouts libres, i.e. $M(C)$ est une immersion d'une réunion de cercles union $j$ segments. Considérons une lentille de ce diagramme (si elle existe). Si on peut la vider par des transformations admissibles (qui fixent les $2 j$ bouts) on a terminé par récurrence sur le nombre d'arêtes. Sinon on recommence le raisonnement avec une lentille du graphe médial associé à $C$. Le nombre d'arêtes de la nouvelle composante $C$ construite diminue strictement chaque fois. La procédure s'arrête donc au bout d'un moment avec un graphe médial $M(C)$ qui possède $2 j^{\prime}$ bouts et qui ne contient aucune lentille. On peut de plus supposer que $\Gamma$ ne se réduit pas à un graphe plus simple par une transformation du type (i), (i'), (ii) ou (ii'). Une vérification de tous les cas possibles montre que ceci se produit seulement avec $j^{\prime}=3$ et on est alors dans le cas de la figure 7 .

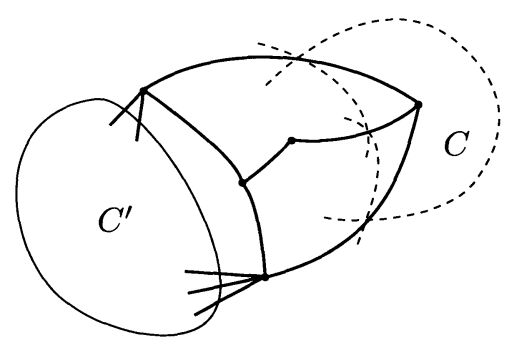

Figure 7.

Le fait qu'on ait $j^{\prime}=3$ implique que $\Gamma \backslash Y$ est composé d'au plus deux composantes : $C$ et éventuellement une autre composante $C^{\prime}$ qui est alors reliée à au plus deux sommets de $Y$. Une inspection de la figure 7 révèle

TOME $123-1995-\mathrm{N}^{\circ} 4$ 
que $\Gamma$ contient alors au moins un sommet de degré 2 ce qui termine la preuve par récurrence sur le nombre d'arêtes de $\Gamma$.

\section{BIBLIOGRAPHIE}

[Bo] Bollobas (B.). - Introduction to graph theory. - Springer, 1979.

[CMM] Curtis (E.), Mooers (E.) and Morrow (J.). - Finding the conductors in circular networks from boundary measurements, Preprint, Washington, 1991, p. 1-31.

[CoV] Colbois (B.) et Colin de Verdière (Y.). - Sur la multiplicité de la première valeur propre d'une surface de Riemann à courbure constante, Comment. Math. Helv., t. 63, 1988, p. 194-208.

[CV1] Colin De Verdière (Y.). - Sur un nouvel invariant des graphes et un critère de planarité, J. Combin. Theory Ser. B, t. 50, 1990, p. $11-20$.

[CV2] Colin de Verdière (Y.). - Réseaux éléctriques planaires I, Comment. Math. Helv., t. 69, 1994, p. 351-374.

[CV3] Colin de Verdière (Y.). - Multiplicités des valeurs propres : laplaciens discrets et laplaciens continus (survey), Rend. Mat. (7), t. 13, 1993, p. 433-460.

[E] EpIfanov (G.V.). - Reduction of a plane graph to an edge by a star-triangle transformation, Soviet Math. Dokl., t. 7, 1966, p. 13-17.

[Gr] Grünbaum (B.). - Convex Polytopes. - Interscience Publishers, London, 1967.

[RST1] Robertson (N.), Seymour (P.) and Thomas (R.). - A survey of linkless embeddings, Preprint, 1991, p. 1-15.

[RST2] Robertson (N.), Seymour (P.) and Thomas (R.). - Linkless embeddings of graphs in 3-space, Bull. Amer. Math. Soc., t. 28, 1993 , p. 84-89.

[vHLS] Van Der Holst (H.), Lovasz (L.) and SchriJver (A.). - Clique minors, graph connectivity, and Colin de Verdière's invariant, Prépublication, 1994, p. 1-11. 